\title{
Discussion of Education Model in Post-pandemic Era and Research on Mobile Learning Application Design Strategy
}

\author{
Yue $\mathrm{CAO}^{1}$, Li-Bin CUI ${ }^{1, *}$, Yong-Fei $\mathrm{YE}^{2}$ and $\mathrm{Yi} \mathrm{LUN}^{2}$ \\ ${ }^{1}$ Graduate School, Hebei North University, Zhangjiakou, Hebei 075000, China \\ ${ }^{2}$ School of Information Science and Engineering, Hebei North University, Zhangjiakou, Hebei 075000, China \\ *Corresponding author. Email: clbzgt@88com.
}

\begin{abstract}
The paper sorts out the evolution of education in China under the influence of the epidemic in 2020, analyzes the advantages and disadvantages of pure online education and offline education, and draws follow conclusions: With current technology, the pure online education model cannot meet the educational requirements due to the lack of real-time, two-way, face-to-face communication. It needs to be combined with the offline education model to form a hybrid education model, and the online education module is designed around the offline education module. On this basis, the paper proposes three types of online education application design strategies: after-school assisted, classroom enhanced and fully live broadcast.
\end{abstract}

Keywords: Mobile learning, Online education, Hybrid education.

\section{INTRODUCTION}

The COVID - 19 has suddenly led to an unprecedented online education practice across the country, and research directions such as mobile education, audio-visual education, and Internet + education have been pushed to the forefront once again. Educational applications have sprung up on major electronic platforms, and nearly a hundred applications compete with each other. However, with the control of the epidemic in my country, schools at all levels and types across the country opened in an orderly manner, and face-to-face teaching is still the main method of education. The various applications that have been savagely grown for half a year are collectively silent at this time. Few applications can break out of the siege and remain vigorous until the post-pandemic era.

Although there are many types of current education apps, their positioning is unclear, their functions are chaotic, and they cannot precisely meet the needs of users. In the end, they are "not easy to use". The paper analyzes the evolution of education in China under the influence of the epidemic in 2020, and tries to answer the following two questions: a) How to develop online education in the postpandemic era? b) What principles should education apps follow for design and development?

In order to determine the research content, according to different learning methods, the current mainstream education mode is divided into pure online education mode, offline education mode and hybrid education mode. Pure online education mode: that is, all communication and information transmission are carried out through the network using electronic equipment; Offline education mode: equivalent to face-to-face teaching, that is, both teachers and students have real-time, two-way communication in the same scene. Hybrid education mode: both online education and face-to-face education are adopted.

\section{THE DEVELOPMENT OF CHINA'S ONLINE EDUCATION MODEL IN 2020}

Affected by the COVID - 19, schools at all levels and types across the country responded to the call of the Ministry of Education to "Suspension of classes without suspending school" in early 2020 and urgently switch to pure online education model. The suddenness of the epidemic and insufficient technical and experience preparations make online education more like a disaster at this time. Every aspect of the environment, equipment, network, and server capacity will expose problems, and the quality of teaching cannot be guaranteed. After the epidemic was brought under control, schools at all levels and all types across the country resumed classes in an orderly manner. After students returned to school, the first examination for students found that students were divided into follow five categories: First, students with a good foundation and strong self-consciousness are the "leaders" in the class, and they dominate the class; Second, students with good foundation and poor consciousness, most of them fall out of the top 10 in the class; Third, students with average foundation and strong self-consciousness who successfully used the holiday to "counterattack"; Fourth, students with average foundation and average selfconsciousness are still in the middle of the class. Fifth, students with poor foundations and poor selfconsciousness have terrible results[1]. 
After a semester of online education practice, it was found that pure online education failed to reach the quality of face-to-face education. Many people questioned online education. For example, at the beginning of the paper Educational Technology as a Learning Path in the Era of Risk Management: Dilemma and Breakthrough, Wu Gang questioned: Is online teaching effective[2]? At this time, most researchers have discovered that there are many longboards in the wooden barrel of online teaching, such as: not limited by space. But there are also fatal shortcomings, such as the "consciousness and selfdiscipline" of students. The current online education is very weak in conscious control. Constructivism believes that knowledge is actively constructed by learners. How can learners who have lost consciousness construct it?

Moreover, although online education is not limited by space, there is no face to face gathering, and there is no expression and movement between each other, and it is difficult to form a real class without being in the same environment. Without classes, it is difficult to use methods such as cooperation and discussion to stimulate learning interest and promote students' deep learning.

In terms of the learning environment, is there really no difference between bedroom study and classroom study? On hardware devices, the blackboard that once covered a wall is now reduced to a screen the size of the palm of your hand. Will the learning experience be better?

After calming down and reflecting on the disadvantages of online teaching, it is not difficult to find that under current technical conditions, pure online education model is not competent for education and can only be used as an auxiliary teaching mode.

After that, the online and offline hybrid teaching model has become a new research direction. Online education model is not restricted in time and space, and face-to-face education model has a huge advantage in the simultaneous interaction of teachers and students. Online or offline education model alone cannot achieve the best results and cannot meet the requirements of today's education. Therefore, for a long period of time in the future, the online and offline hybrid education model will be the mainstream education model.

There is also a controversy. When $5 \mathrm{G}$ technology is popularized, the problem of network stuck will be completely solved; and with the advancement of virtual reality technology, augmented reality technology, holographic technology and video conferencing technology, scenes in real classrooms are expected to be virtualized. So that everyone can truly feel that online teaching is as realistic as face-to-face teaching. By then, most of the disadvantages of online interaction will no longer exist[3]. This statement is analyzed from a technical point of view. However, can these technologies really show people's every move perfectly? Is it possible to replicate the interactive feedback between humans and objects? Assuming that the network speed meets the requirements, can the refresh rate, resolution, and other parameters of the device meet the requirements? From an economic point of view, Premier Li Keqiang mentioned that there are 600 million people in China with a monthly income of less than 1,000 yuan. When these technologies mature, how long will it take to appear on campus? How long will it take to be popularized? Can the price of the equipment be affordable to most people? Perhaps the advancement of science and technology is rapid, but education is also subject to economic constraints. Economic development has its own laws, and these devices may not be popular in a short period of time.

In addition, there is another issue that deserves attention: the carnival of technology and the barbaric growth of online education make people ignore the essence of education. All the purpose of education is to teach and educate people, online and offline are just means. In the education process, the communication between teachers and students is very important. Communication in education is not only the transmission of information, but also the infection of emotions, attitudes, and values. After the isolation of technology, communication between teachers and students becomes blurred, it is difficult to convey emotions between teachers and students. Students cannot feel the enthusiasm of teachers, teachers cannot perceive the state of students, and a lively person is more like a high-level artificial intelligence under the isolation of technology. The emotions, attitudes, and values prepared in the lesson plans cannot be reflected, making the classroom a mere indoctrination of knowledge.

Face-to-face education model benefits from the advantages of simultaneous communication between teachers and students. It can not only transfer knowledge, organize groups for cooperation, discussion, and confrontation, but also infect students with enthusiasm and cause students to change their emotions, attitudes and values. A closer teacher-student relationship and a more harmonious learning atmosphere are conducive to the implementation of education. This unshakable absolute advantage makes offline education a central position in the hybrid education model. Some schools have adopted the classroom + MOOC model and achieved good results. Some students will take the initiative to find suitable online courses while participating in school courses. Therefore, in the mixed education model, face-to-face modules should be the center, and online modules should be designed and expanded around face-to-face modules.

\section{EDUCATIONAL APPLICATION DESIGN STRATEGY BASED ON HYBRID EDUCATION MODEL}

Based on the above discussion, a hybrid education model with offline modules as the center and online modules assisting offline modules can be determined. In this mode, according to whether there are face-to-face, same-scene, and real-time two-way communication between teachers and students, the paper proposes three types of education application design strategies: after-school assisted, classroom enhancement, and full live broadcast. 


\subsection{After Class Assisted}

After-school-assisted education applications do not have face-to-face, real-time two-way communication between teachers and students, such as voice calls and live images. This category is only for after-school assisted, and aims to help students improve their learning efficiency after school. It does not involve classrooms and live broadcasts, and does not have any synchronous communication methods. It can be equipped with soft communication methods, that is non-immediate and asynchronous communication methods such as forums, messages, private messages, and e-mails. From this perspective, video courses such as major MOOCs and micro-classes should belong to the after-school assisted type.

The after-school assistance type can also design some functions to free students and teachers from repetitive routine tasks such as sending and receiving homework and judging homework. The core purpose of after-class assisted applications is to help teachers control students' under-class status through technology and improve students' learning efficiency.

It should be noted that the principle of after-school assisted is that the tasks can be done now or later, but they need to be completed before the deadline. Tasks can be arranged by teachers, or students can choose freely according to their own situation. It is a learning method that exists outside of the classroom and is used as a supplement to the classroom to enrich students' spare time. However, corresponding to the state in class, it is free and autonomous after class, and students can adjust tasks appropriately according to their own situation, and there is more space for free arrangement.

\subsection{Classroom Enhancement}

Current technology cannot fully simulate face-to-face communication scenarios. Therefore, the core competitiveness of offline classrooms lies in having a face-to-face, same-scene, and real-time communication environment. The micro movements, expressions and tone of teachers and students will be seen by everyone in the class. The teachers and students in the same environment form a collective, the class. All students and teachers are one of them, and can use various teaching methods such as discussion, cooperation, debate, confrontation. A closer teacher-student relationship and a more harmonious learning environment help teachers and students to communicate emotionally and attitude. Based on this characteristic, all functions of this classification should be designed around offline classrooms, aiming to enrich the classroom expression and enhance the classroom teaching effect. The core idea of classroom enhancement is to enrich the classroom based on the characteristics of the communication between the people based on face-to-face. Therefore, all the functions currently used in the classroom can be regarded as classroom enhancements, such as multimedia courseware.
Classroom enhancements are designed around offline classrooms, and there is no communication method. All communication is carried out in an offline manner to expand the functions of the classroom, enrich the content taught by teachers, help students understand, and improve teaching effects. The class here is not necessarily a traditional chalk blackboard class, it can be a laboratory. Thanks to the exploration of audio-visual education, there are more and more mature applications of classroom enhancements. However, with the advancement of technology, traditional multimedia and slideshows have gradually failed to meet the requirements, and better applications have not yet become popular.

It should be noted that although offline classrooms have absolute advantages in communication, they also have their limitations. The direction of the use of technology should be to break the limitations of offline classrooms. This is the core advantage of the hybrid education model, instead of focusing on the perfect presentation of the communication scene.

\subsection{Full Live Broadcast}

The fully live broadcast type is an online classroom with complete online teaching functions. It is a software made in cyberspace, which can ensure the synchronous two-way communication between teachers and students in cyberspace, and conduct lectures in cyberspace. It has complete classroom functions for emergency use. Although the teaching effect of the pure online education model is difficult to meet the requirements, the global epidemic situation is not optimistic, the domestic epidemic situation is repeated occasionally, the vaccination rate is less than 10\% (as of April 2021), and the future trend is unpredictable. In order to prevent the suspension of classes caused by the rebound of the epidemic in some areas, pure online classes still have their meaning.

And in the long run, the development of 5G technology, virtual reality technology, and remote conference technology will continue to narrow the gap between online communication and offline communication. The impact of technology on education can be disruptive. Many researchers are imagining the design and exploration of online education in the future, but the current live classroom is still based on the model of offline classrooms, or even directly copying offline classrooms, and has not achieved satisfactory results. The advantages of technology have not been fully utilized, so the exploration of the pure online classroom model cannot be given up.

\section{CONCLUSION}

The controversy over online education has not stopped. Many researchers are trying to subvert the education industry with technology according to their own ideas. However, technological imperfections and economic constraints make the ideal online education still far away from us. The advantages of face-to-face education cannot be replaced for a long time in the future. In the hybrid 
education model, the applications we design must be accurately positioned, the use scenarios must be clarified, the problems and pain points in education must be identified, don't be obsessed with technology and neglect education. If the new idea cannot effectively solve certain problems, it can only be eliminated in the end.

After the above discussion, it can be concluded that under the current technological and economic environment, the blind pursuit of "high-tech" and "good equipment" does not match the needs of teachers and students. In the mixed education model, face-to-face education is the core, and the use of existing technology and equipment to strengthen the effect of face-to-face education meets the objective requirements and that is the mainstream in the future. Based on this, the paper further proposes three types of educational application design principles: after-school assisted, classroom enhanced and fully live broadcast, to help various educational applications locate accurately and meet the needs of teachers and students. Fully cover all kinds of scenarios.

\section{REFERENCES}

[1] P. Xu, J. Cao, et. al., Quantum chemical study on the adsorption of megazol drug on the pristine BC3 nanosheet, Supramolecular Chemistry, 33(2021)63-69.

[2] Wu Gang, Educational Technology as a Learning Path in the Era of Risk Management: Dilemma and Breakthrough, J. CSSCI. Open Education Research,2020,26(03):11-25.

[3] WANG Zhuli, Replacing the Classroom or Going Beyond It?_-Debates and Reflections on Online Education, J. CSSCI. Modern Distance Education Research,2020,32(05):35-45.

[4] P. Xu, N. Na, A. M. Mohamad, Investigation the application of pristine graphdiyne (GDY) and borondoped graphdiyne (BGDY) as an electronic sensor for detection of anticancer drug, Computational and Theoretical Chemistry, 1190(2020): 112996.

[5] Y. Du, L. Song, C. Liu, J. Fu, J. Sun, Y. Tang, A bibliometric analysis of research on chimeric antigen receptor T cells, Cancer Cell Research,7(2020)704-713.

[6] R. Shen, Diagnosis and Video-Assisted Thoracic Surgery of Pulmonary Sclerosing Pneumocytoma, Cancer Cell Research,7(2020)671-675. 